\title{
Interoceptive Functioning in Schizophrenia and Schizotypy
}

\author{
Lénie J. Torregrossa $\mathrm{a}^{{ }^{*}}$ \\ Amad Amedy ${ }^{\mathrm{a}}$ \\ Jacqueline Roig ${ }^{\mathrm{a}}$ \\ Andrea Prada ${ }^{\mathrm{a}}$ \\ Sohee Park ${ }^{\mathrm{a}}$
}

${ }^{\text {a }}$ Department of Psychology, Vanderbilt University, Nashville, TN, USA.

*Correspondence concerning this article should be addressed to Lénie Torregrossa

11121 st Ave. S, Wilson Hall, Room 311 Vanderbilt University, Nashville, TN, 37240

lenie.j.torregrossa@vanderbilt.edu ; (615) 322-3435 


\begin{abstract}
Though bodily self-disturbances are well documented in schizophrenia, interoceptive functioning (i.e., the perception of the internal state of the body) remains poorly understood in this population. In fact, only two studies to date have empirically measured interoceptive ability in schizophrenia. Both studies documented a deficit in interoceptive accuracy (i.e., objective performance on a heartbeat detection task), and one noted differences in interoceptive sensibility (i.e., subjective experience of interoception) in this population. To our knowledge, interoceptive awareness (i.e., metacognitive awareness of one's interoceptive ability) has never been measured in schizophrenia and the link between interoceptive functioning and schizotypy remains unexplored.
\end{abstract}

The present study addresses this gap by investigating the three dimensions of interoception in individuals with schizophrenia and matched controls (Experiment 1, N=58) and in relation to schizotypal traits (Experiment 2, $\mathrm{N}=109$ ).

Consistent with the literature, Experiment 1 documented a deficit in interoceptive accuracy and differences in interoceptive sensibility in individuals with schizophrenia. For the first time, our study revealed intact interoceptive awareness in individuals with schizophrenia. Against our expectations, we found no link between schizotypy and interoceptive functioning in Experiment 2.

Our novel findings bear important clinical implications as insight into one's interoceptive limitations (i.e., intact interoceptive awareness) might promote treatment seeking behavior in schizophrenia. The lack of association between interoceptive ability and schizotypy in non-helpseeking youths suggests that changes in interoception may only arise with the onset of psychosis. 
Keywords: interoception, schizophrenia, schizotypy, bodily-self, self-disturbances 


\section{INTRODUCTION}

Throughout the course of illness, self-disturbances are central to schizophrenia (Sass and Parnas, 2003; Nelson et al, 2012; Park and Nasrallah, 2014). The implicit awareness of our body as a unified entity with clear boundaries allows us to distinguish the self from the environment, thereby scaffolding adaptive social interactions (Palmer \& Tsakiris, 2018). In schizophrenia, this basic sense of the bodily self is disrupted (Bleuler, 1911; Sass and Parnas 2003). Narratives of aberrant self experiences (e.g., Kean 2009; Saks, 2007) are supported by contemporary empirical evidence documenting anomalous body ownership, agency, self-identification and emotional embodiment in this population (Ferroni et al., 2019; Hur et al., 2014; Raballo et al, 2021; Sandsten et al., 2020; Thakkar et al, 2011; Torregrossa et al., 2019). Importantly, such anomalous self-experiences predate schizophrenia onset (Nelson et al., 2012) and lead to social impairments and disconnection (Michael and Park, 2016). Thus, elucidating the mechanisms underlying self-disturbances in schizophrenia could lead to more effective treatments.

Interoception is central to the experience of a unified bodily self (Tsakiris, 2017). Interoception refers to the awareness of sensations and physiological signals originating from inside the body (Craig, 2002). Beyond its role in homeostasis (Quigley et al., 2021), interoception is central to the awareness of our own bodily sensations, which is essential to the sense of bodily self (Damasio, 2012). Interoception has also been shown to play a central role in self-recognition (Filippetti and Tsakiris, 2017, Tajadura-Jimenez and Tsakiris, 2014), self-other distinction (Palmer and Tsakiris, 2018), peripersonal space (Ardizzi and Ferri, 2018), and body ownership (Suzuki et al., 2013; Tsakiris et al., 2011). Thus, interoception and sense of self are intricately twined. 
Three distinct aspects of interoception can be captured empirically (Garfinkel et al., 2015): (1) interoceptive accuracy (i.e., the objective performance on behavioral tasks assessing the ability of a participant to perceive their internal signals), (2) interoceptive sensibility (i.e., the subjective evaluation of one's own interceptive ability), and (3) interoceptive awareness (i.e., the metacognitive awareness of one's interoceptive ability). Interoceptive accuracy and awareness are typically studied by examining one's awareness of their heartbeats. Heartbeat counting tasks typically require participants to count of the number of heartbeats occurring during a defined temporal interval or to detect the synchronicity between their heartbeats and an external stimulus (Garfinkel et al., 2015). Given its subjective nature, interoceptive sensibility is assessed using self-report instruments (Garfinkel et al., 2015).

Although interoception is central to the sense of bodily self, and self-disturbances are core features of schizophrenia, interoception has not been systematically investigated in this population. To date, only two studies have experimentally investigated interoception in schizophrenia (Ardizzi et al., 2016; Koreki et al., 2020). Both studies found reduced interoceptive accuracy in schizophrenia, which was linked to the severity of positive symptoms. In addition, recent studies documented altered interoceptive sensibility in individuals with schizophrenia (Koreki et al., 2020) and high-risk individuals (Barbato et al., 2021). To our knowledge, interoceptive awareness has never been measured in schizophrenia.

Interoception has also never been studied in relation to schizophrenia liability in the general population. Schizotypy was first introduced as a set of inherited personality traits that yields a vulnerability for schizophrenia (Meehl, 1962). Beyond the realm of the familial risk, schizotypy broadly refers to the set of personality traits and experiences related to subthreshold psychotic experiences in the general population (Debbané et al., 2015). Schizotypy is 
multidimensional, and its factors are analogous to the three major symptom clusters in schizophrenia: positive, negative, and disorganized (Raine et al., 1994). Though the nature of the schizotypy construct remains debated, there is a broad consensus that schizotypy may be dimensional and is associated with schizophrenia liability (Grant et al., 2015). In the second part of this study, we therefore use schizotypy to investigate interoception in a population that presents qualitatively similar to, and may have latent liability for, schizophrenia.

The present study addresses the gaps previously mentioned by investigating the three dimensions of interoception (i.e., interoceptive accuracy awareness, and sensibility) in a group of individuals with schizophrenia (Experiment 1) and in individuals with varying levels of schizotypy (Experiment 2).

\section{EXPERIMENT 1: INTEROCEPTIVE ACCURACY, AWARENESS AND SENSIBILITY IN SCHIZOPHRENIA AND CONTROL GROUPS}

We investigated interoceptive accuracy, awareness, and sensibility in individuals with schizophrenia (SZ) and demographically matched control participants (CO). Based on previous studies (Ardizzi et al., 2016; Koreki et al., 2020), we expected to find reduced interoceptive accuracy and altered interoceptive sensibility in SZ. We did not have an a-priori hypothesis for interoceptive awareness in SZ.

\section{1. METHODS}

\section{1. 1. Participants}


Thirty individuals who met the DSM-5 criteria for schizophrenia $(\mathrm{N}=18)$ or schizoaffective disorder $(\mathrm{N}=12)$ were recruited from an outpatient facility in Nashville, TN (SZ). All SZ participants were taking antipsychotic medication. Twenty-eight $\mathrm{CO}$ with no history of DSM-5 disorders were recruited from the same community by advertisements. Diagnoses were made using the Structured Clinical Interview for DSM-5- Research Version (First et al., 2015). Potential participants who met DSM-5 criteria for schizophrenia or schizoaffective disorder and had no comorbidities were included in the SZ group, while those who did not meet criteria for any past or current DSM-V disorders and reported no family history of psychotic disorders were included in the CO group. SZ symptoms were assessed with the Brief Psychiatric Rating Scale (BPRS, Version 4.0; Ventura et al.,1993), the Scale for the Assessment of Positive Symptoms (SAPS, Andreasen, 1984), and the Scale for the Assessment of Negative Symptoms (SANS, Andreasen, 1983). Exclusion criteria for both groups were substance use or alcohol abuse within the past 6 months, brain injury, neurological disease, and IQ $<70$. The two groups were balanced on gender, age, and handedness. The Institutional Review Board of Vanderbilt University approved study protocols, and written informed consent was collected from each participant. Participants were compensated for their time. Demographic, self-report, and clinical information for Experiment 1 is presented in Table 1.

Table 1. Demographic, self-report, and clinical information for Experiment 1 participants.

\begin{tabular}{lllll}
\hline & $\begin{array}{l}\mathrm{SZ}(\mathrm{N}=30) \\
\text { Mean }(\mathrm{SD})\end{array}$ & $\begin{array}{l}\mathrm{CO}(\mathrm{N}=28) \\
\text { Mean }(\mathrm{SD})\end{array}$ & Test statistic & $p$ \\
\hline Gender $(\mathrm{M} / \mathrm{F})$ & $16 / 14$ & $14 / 14$ & $\chi^{2}=0$ & $p=1$ \\
Age & $47.93(10.21)$ & $48.68(8.2)$ & $t=0.31$ & $p=0.76$
\end{tabular}




\begin{tabular}{|c|c|c|c|c|}
\hline $\begin{array}{l}\text { Handedness } \\
\text { (R/L/Both) }\end{array}$ & $24 / 5 / 1$ & $26 / 2 / 0$ & $\chi^{2}=2.30$ & $p=0.32$ \\
\hline Estimated IQ ${ }^{\mathrm{a} *}$ & $101.96(9.96)$ & $108.61(9.13)$ & $t=2.65$ & $p=0.01$ \\
\hline Perceived Stress ${ }^{b *}$ & $18.83(7.83)$ & $12.68(7.15)$ & $t=-3.13$ & $p=0.003$ \\
\hline Loneliness $^{\mathrm{c} *}$ & $40.50(11.85)$ & $33.71(8.48)$ & $Z=-2.34$ & $p=0.02$ \\
\hline $\begin{array}{l}\text { Alexithymiad }^{\mathrm{d}} \\
\text { DDF* }^{*} \\
\text { DIF** }^{*} \\
\text { EOT* }^{*}\end{array}$ & $\begin{array}{l}12.77(4.41) \\
15.97(7.58) \\
20.57(74.75)\end{array}$ & $\begin{array}{l}9.07(2.60) \\
9.79(3.45) \\
17.68(4.02)\end{array}$ & $\begin{array}{l}Z=-3.49 \\
Z=-3.32 \\
t=-2.50\end{array}$ & $\begin{array}{l}p=0.0005 \\
p=0.0009 \\
p=0.02\end{array}$ \\
\hline $\begin{array}{c}\text { Current Affect } \\
\text { Positive* }^{\mathrm{e}} \\
\text { Negative }\end{array}$ & $\begin{array}{l}33.36(8.91) \\
21.33(11.10)\end{array}$ & $\begin{array}{l}39.18(6.37) \\
16.46(6.03)\end{array}$ & $\begin{array}{l}t=2.74 \\
Z=-1.62\end{array}$ & $\begin{array}{l}p=0.008 \\
p=0.10\end{array}$ \\
\hline BPRS & $19.90(9.74)$ & - & - & - \\
\hline SAPS & $24.37(16.62)$ & - & - & - \\
\hline SANS & $38.47(17.84)$ & - & - & - \\
\hline CPZE dose $(\mathrm{mg} / \text { day })^{\mathrm{f}}$ & $533.68(768.87)$ & - & - & - \\
\hline Illness duration (years) & $26.24(7.61)$ & - & - & - \\
\hline $\begin{array}{l}\text { Number of } \\
\text { hospitalizations }\end{array}$ & $7.93(8.27)$ & - & - & - \\
\hline
\end{tabular}

* Significant difference between SZ and CO

${ }^{\text {a }}$ Estimated from the National Adult Reading Test, Revised (NART-R; Blair and Spreen, 1989).

${ }^{\mathrm{b}}$ Measured using the Perceived Stress Scale (PSS; Cohen et al., 1983).

${ }^{\mathrm{c}}$ Measured using the UCLA Loneliness scale, Version 3 (Russell, 1996).

${ }^{\mathrm{d}}$ Measured using the TAS-20 (Bagby, Parker, and Taylor, 1994). DDF= Difficulty Describing Feelings; DIF=

Difficulty Identifying Feelings; EOT= Externally Oriented Thinking.

${ }^{\mathrm{e}}$ Measured using the Positive and Negative Affect Schedule (PANAS; Watson et al., 1988).

${ }_{\mathrm{f}}^{\mathrm{f}}$ Antipsychotic dosage was converted to chlorpromazine equivalent (CPZE; Andreasen et al., 2010).

\section{1. 2. Interoception Measures}

2. 1. 2. 1. Heartbeat Counting Task (Schandry, 1981)

The HCBT was used to measure interoceptive accuracy and awareness. In this task, participants were instructed to sit with their hands on their lap facing up to prevent facilitation of 
heartbeat detection. They were instructed to "count the number of times [you] think [your] heart beats from the time I say start to the time I say stop" while a Bluetooth Polar H10 heartrate monitor (Polar Electro Öy, Kempele, Finland) measured their actual heart beats. Heart rate data was transmitted to a mobile device via Bluetooth using the Polar Beat App. Participants completed a practice trial and four test trials of varying lengths (i.e., 33s, 25s, 41s, 17s). After each trial, participants were asked "how many times did your heart beat?" and "how confident are you that your guess is correct, on a scale from zero to ten?"

Interceptive accuracy was computed as follows: $1 / 4 \Sigma(1-(\mid$ recorded -counted|) / recorded $)$ (Schandry et al., 1981). Higher scores represent less discrepancy between counted and recorded heartbeats, therefore indicating higher interoceptive accuracy.

Performance on the HBCT is influenced by a number of factors (i.e., Knapp-Kline and Kline, 2005; Ring and Brener, 1996; Rouse et al., 1988). Thus, participants' Body Mass Index (BMI), resting heart rate (i.e., average heart rate recorded during the HBCT), knowledge of their resting heart rate (i.e., absolute difference between estimated and measured resting heartrate), and heart rate variability (HRV, measured as the root mean square of successive RR interval differences; Shaffer and Ginsberg, 2017), were assessed and included as covariates in the interoceptive accuracy analyses. In addition, one of the major criticisms of the HBCT is that participants may be estimating the number of seconds elapsed rather than counting their heartbeat. In line with other studies (i.e., Ainley et al., 2014; Filippetti and Tsakiris, 2017), we therefore asked participants to estimate the duration of four time intervals (i.e., 33s, 25s, 41s, 17s) and entered time perception accuracy as a covariate in the subsequent analyses.

Interoceptive awareness was computed by calculating the Spearman correlation coefficient between the interoceptive accuracy and the self-reported confidence rating across 
trials for each individual (Garfinkel et al., 2015). Interoceptive awareness scores therefore varied between -1 and 1 , with higher score indicating greater correspondence between interoceptive accuracy and confidence ratings, therefore indicating higher interoceptive awareness.

\section{1. 2. 2. Multidimensional Assessment of Interoceptive Awareness (Mehling et al., 2012)}

The MAIA was used to assess interoceptive sensibility. The MAIA yields eight subscales: 1) Noticing refers to one's awareness of their bodily sensations, 2) Not-Distracting measures one's tendency not to ignore sensations of discomfort or pain, 3) Not-Worrying assesses an individual's tendency not to experience emotional distress in response to uncomfortable or painful bodily sensations, 4) Attention Regulation refers to one's ability to sustain and control attention to body sensations, 5) Emotional Awareness refers to the awareness of the connection between one's bodily sensations and their emotional states, 6) Self-Regulation measures one's ability to regulate distress by paying attention to their body sensations, 7) Body Listening assesses one's tendency to use bodily sensations for insight and decision-making, 8) Trusting measures one's tendency to experience one's own body as safe and trustworthy. Scores for each subscale are averaged. Higher scores indicate higher interoceptive sensibility across subscales. The MAIA has demonstrated good psychometric properties (Mehling et al., 2012) and is one of the most widely used instruments measuring interoceptive sensibility.

\section{1.3. Procedure}

After informed consent, participants completed the interoception measures (i.e., HBCT and MAIA) as well as self-report questionnaires measuring alexithymia (TAS-20; Bagby, Parker, and Taylor, 1994), current affect (PANAS; Watson et al., 1988), perceived stress (PSS, Cohen et 
al., 1983), and loneliness (UCLA Loneliness scale, Version 3; Russell, 1996). The order in which participants completed the HBCT, the MAIA, self-reported measures, cognitive measures (i.e., NART) and clinical interviews (i.e., SAPS, SANS, BPRS; for SZ only) was randomized. All of the self-report measures used have demonstrated good psychometric properties including (at least) satisfactory reliability and validity.

\section{1. 4. Data Analysis}

Statistical analyses were implemented in R (Version 3.5.1, R Core Team, 2018). Due to the presence of outliers, a robust regression model with M estimator was used to assess the group difference in interoceptive accuracy. The model included group $(\mathrm{SZ}=1, \mathrm{CO}=0)$, the HBCT control variables (i.e., age, HR knowledge, resting HR, HRV, BMI, and time perception accuracy) and self-report measures (i.e., perceived stress, loneliness, alexithymia, affect) as predictors. The group difference in interoceptive awareness was assessed using a Mann-Whitney test. A MANOVA was used to examine group differences in interoceptive sensibility and posthoc univariate analyses were conducted to assess group differences in each of the eight dimensions of interoceptive sensibility. Finally, the relationship between interoceptive ability (i.e., interoceptive accuracy, awareness, sensibility) and demographic (i.e., gender, age) and clinical variables (i.e., symptoms severity, medication dosage) was assessed using correlation analyses, Mann-Whitney, $t$-tests, and MANCOVA. 


\section{2. RESULTS}

\section{2. 1. Self-report measures}

Significant group differences were found across self-report measures. SZ reported feeling more stressed $\left(M_{s z}=18.83, S D s z=7.83, M_{c o}=12.68, S D c o=7.15, t=-3.13, p=0.003, d=-0.82\right)$ and lonelier $\left(\right.$ Median $_{\mathrm{sz}}=42.5$, Median $\left._{\mathrm{co}}=31.5, Z=-2.34, p=0.02, r=-0.31\right)$ than CO. SZ also reported higher levels of alexithymia on all three TAS-20 subscales. Indeed, SZ reported higher Difficulty Describing Feelings $\left(\operatorname{Median}_{\mathrm{sz}}=12.5\right.$, Median $\left._{\mathrm{co}}=9.0 Z=-3.49, p=0.0005, r=-0.46\right)$, Difficulty Identifying Feelings $\left(\right.$ Median $_{\mathrm{sz}}=15.5$, Median $\left._{\mathrm{co}}=8.0, Z=-3.32, p=0.0009, r=-0.44\right)$, and Externally Oriented Thinking $\left(M_{s z}=20.57, S D s z=74.75, M_{c o}=17.68, S D c o=4.02, t=-2.50, p=0.02\right.$, $d=-0.65)$. Finally, SZ reported significantly lower levels of PANAS-positive affect $\left(M_{s z}=33.63\right.$, $\left.S D s z=8.91, M_{c o}=39.18, S D c o=6.37, t=2.74, p=0.008, d=0.71\right)$, but no group difference was found on PANAS-negative affect $(Z=-1.62, p=0.10)$.

\section{2. 2. Interoceptive Accuracy}

A robust regression model revealed a significant deficit in interoceptive accuracy in SZ $(M=0.48, S D=0.25)$ compared to $\mathrm{CO}(M=0.73, S D=0.19)$, after controlling for age, resting $\mathrm{HR}$, HR knowledge, HRV, BMI, and time perception accuracy, $t=-2.29, p=0.03$. See Fig. 2. 


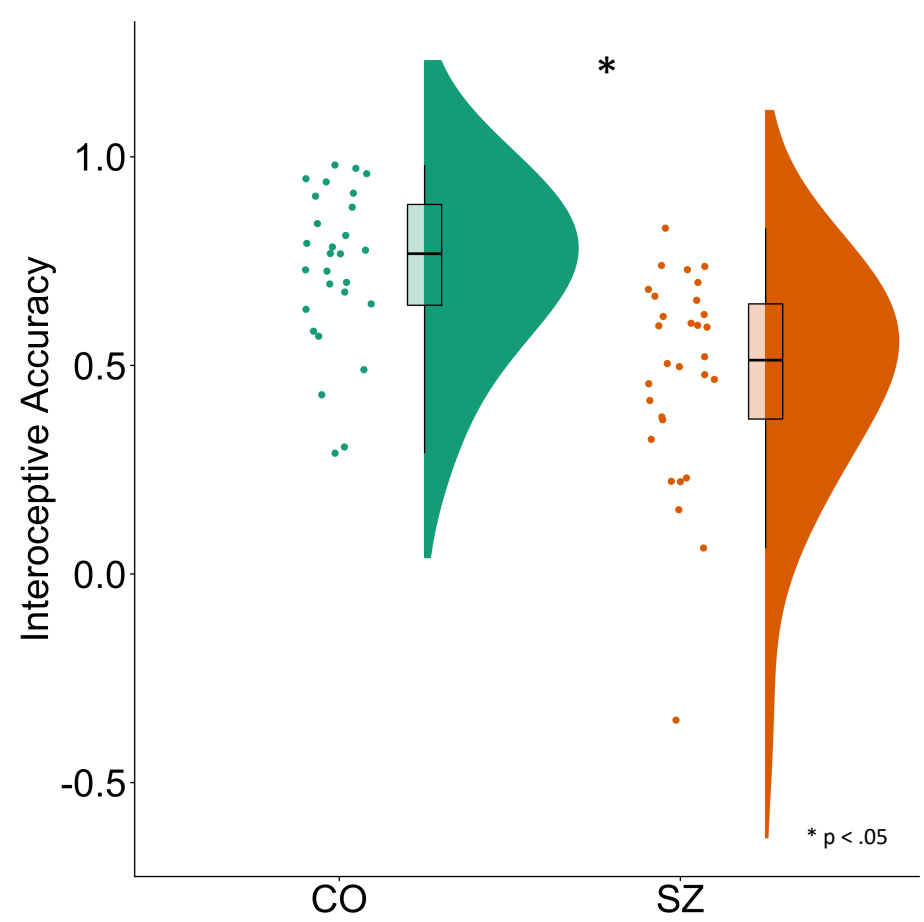

Fig. 2. Raincloud plots of Interoceptive Accuracy in SZ and CO

Time perception accuracy was also found to significantly predict interoceptive accuracy, $t=2.39, p=0.02$. Notably, a trending group difference in time perception accuracy was found $(Z=-$ $1.9, p=0.054)$, suggesting that $\mathrm{SZ}(M=-0.67, S D=7.44)$ might have difficulty with time perception compared to $\mathrm{CO}(M=0.58, S D=1.18)$. None of the other control (i.e., resting HR, HR knowledge, HRV, and BMI) or self-reported (i.e., stress, loneliness, alexithymia and current affect) variables were found to predict interoceptive accuracy (all $p$ 's $>0.05$ ). A significant group difference was found in HR knowledge, such that the difference between estimated and measured HR was greater in $\mathrm{SZ}(M=39.02, S D=24.40)$ than $\mathrm{CO}(M=16.04, S D=12.72), Z=-3.76, p=0.0002$, indicating a deficit in HR knowledge in SZ. SZ $(M=33.47, S D=7.92)$ were also found to have significantly higher BMI than $\mathrm{CO}(M=28.69, S D=5.96), Z=-2.26, p=0.02$. No group difference was found in resting HR or HRV. 


\section{2. 3. Interoceptive Awareness}

Interoceptive awareness was not found to significantly differ between SZ $(M=0.16$, $S D=0.51)$ and $\mathrm{CO}(M=0.21, S D=0.51), Z=-0.49, p=0.62$ (see Fig. 3).

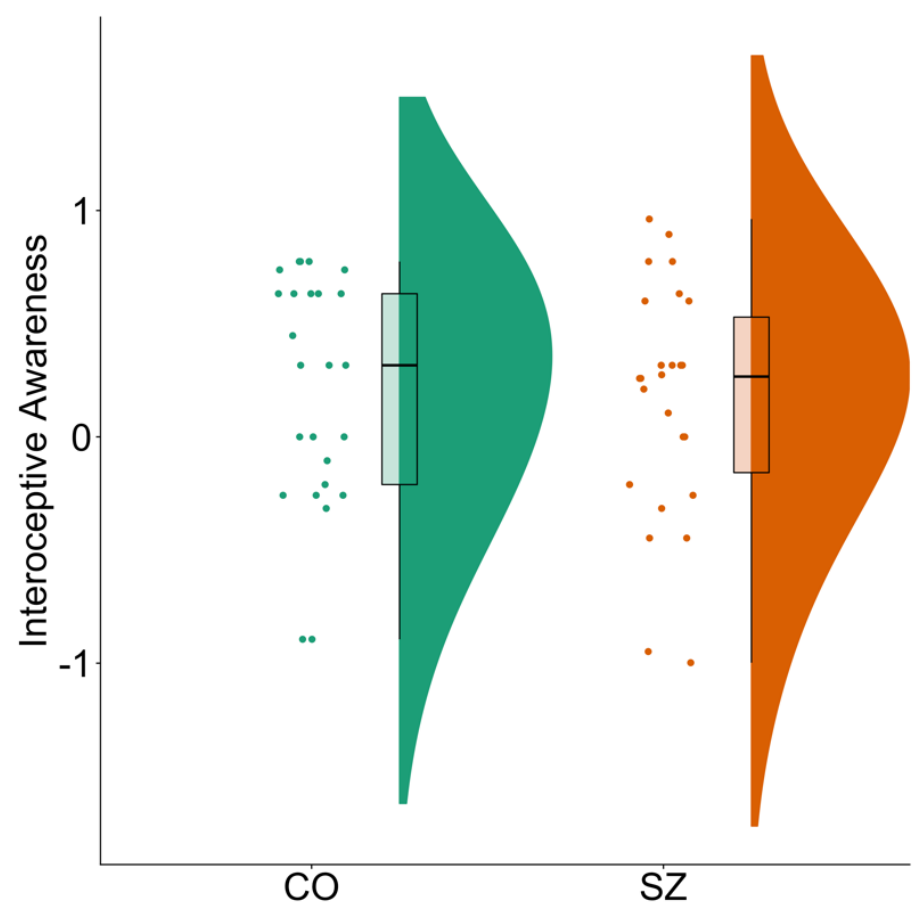

Fig. 3. Raincloud plots lot of Interoceptive Awareness in SZ and CO

\section{2. 4. Interoceptive Sensibility}

A MANCOVA revealed a significant group difference in interoceptive sensibility, $F(8,49)=2.21, p=0.04$. Post-hoc univariate analyses showed that Attention Regulation, $F(1,56)=6.44, \mathrm{p}=0.01, \eta^{2}=0.10$, Not Worrying, $F(1,56)=3.97, \mathrm{p}=0.05, \eta^{2}=0.07$, and Trusting, $F(1,56)=6.56, \mathrm{p}=0.01, \eta^{2}=0.10$, were significantly lower in SZ than CO. Conversely, SZ scored significantly higher on Not Distracting than $\mathrm{CO}, F(1,56)=4.75, \mathrm{p}=0.03, \eta^{2}=0.08$. SZ and CO did 
not significantly differ in Body Listening, Emotional Awareness, Noticing, and Self-Regulation (all $p$ 's $>0.05)$. See Fig. 4.

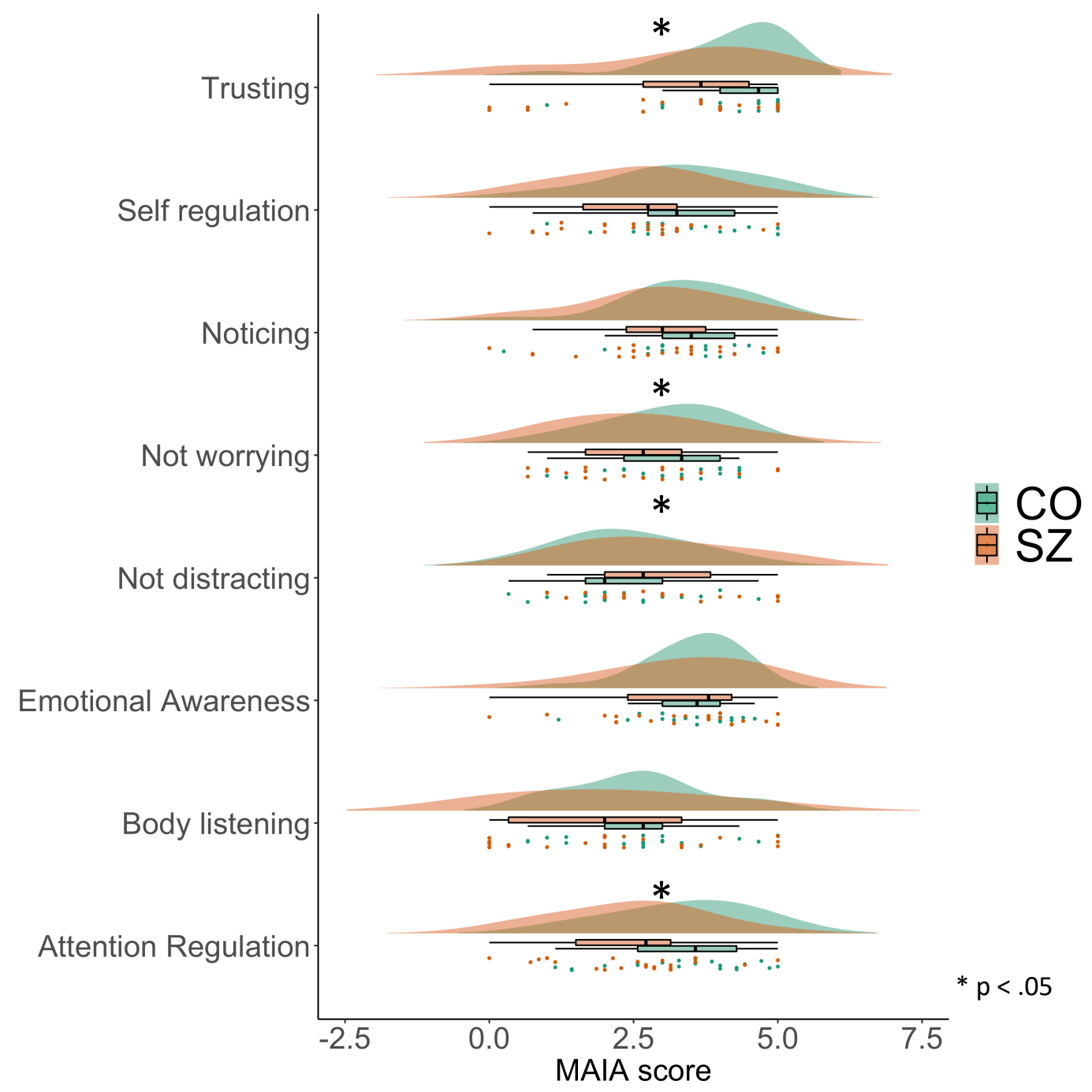

Fig. 4. Raincloud plots of Interoceptive Sensibility in SZ and CO

\section{2. 5. Interoceptive ability and demographic and clinical variables}

Regression analyses revealed no link between symptom severity (i.e., overall psychiatric symptoms, positive symptoms, negative symptoms) and interoceptive ability (i.e., accuracy, 
awareness, sensibility) in SZ (all $p$ 's $>0.05$ ). Medication dosage was not correlated with interoceptive accuracy $(r(28)=-0.21, p=0.29)$, though it was negatively correlated with interoceptive awareness $(r(28)=-0.44, p=0.04)$. A regression analysis revealed that the Emotional Awareness factor of the MAIA predicted antipsychotic medication dosage $(\beta=0.99, p=0.01)$. No relationship was found between medication dosage and other aspects of interoceptive sensibility (all $p$ 's $>0.05$ ).

Spearman correlation analyses revealed that age was not significantly correlated with interoceptive accuracy $(r(56)=-0.01, p=0.94)$, interoceptive awareness $(r(56)=-0.02, p=0.87)$, or interoceptive sensibility (all $p$ 's $>0.10)$. Similarly, interoceptive accuracy $(Z=-0.22, p=0.82)$, interoceptive awareness $(Z=-1.27, p=0.21)$, and interoceptive sensibility $(F(8,49)=0.72, p=0.67)$ did not significant differ between genders.

\subsection{DISCUSSION}

Consistent with previous studies, we found evidence for impaired HBCT performance in SZ. Across the literature, SZ's average interoceptive accuracy was estimated at $0.32 \pm 0.34$ (Koreki et al., 2020), $0.37 \pm 0.63$ (Ardizzi et al., 2016) and $0.48 \pm 0.25$ (present study). Though largely consistent, we note that the SZ sample in our study performed marginally better than in the previous two studies. This difference might be due to the fact that our sample exclusively included outpatients, while Koreki et al.'s study (2020) included inpatient individuals. Interestingly, though interoceptive accuracy has been shown to decline with age (Khalsa et al., 2009) the older SZ in our study (age $=47.93 \pm 10.21$ ) were found to have higher interoceptive accuracy than in Ardizzi et al.'s study (age $=33.78 \pm 6.33$ ). We also note that the average illness 
duration in our sample (26.24 \pm 7.61$)$ was higher than that reported by Ardizzi et al. (2016) (9.22 \pm 3.61 ). Altogether, these findings suggest that interoceptive accuracy deficits are present throughout the course of schizophrenia illness, though they might be more severe in the early/acute stages. This remains to be tested empirically.

Against our predictions, there was no link between interoceptive accuracy and the severity of symptoms. Though the two previous studies documented a link between interoceptive accuracy and overall positive symptoms (Ardizzi et al., 2016; Koreki et al., 2020), the results were inconsistent at the item level. In fact, Ardizzi et al. (2016) reported a relationship between interoceptive accuracy and grandiosity, while Koreki et al. (2020) noted that the severity of conceptual disorganization and hallucinations (as well as negative symptoms) predicted interoceptive deficits. At present, the relationship between interoceptive accuracy deficits and schizophrenia symptomatology remains unclear.

There was no group difference in interoceptive awareness, the metacognitive awareness of one's performance on the HBCT. SZ's intact interoceptive awareness suggests insight into their low interoceptive accuracy. Importantly, medication dosage was negatively correlated with interoceptive awareness, suggesting that antipsychotic medication might dampen insight into interoceptive deficits. However, it is important to note that interoceptive awareness was intact in SZ regardless of medication dose. To our knowledge, our study is the first to empirically explore interoceptive awareness in schizophrenia. Thus, our findings provide preliminary evidence of intact interceptive awareness in this population. Intact interoceptive awareness in SZ is noteworthy especially since metacognitive deficits are documented in schizophrenia (Lysaker and Dimaggio, 2014). We note that the interpretation of this finding is limited by our relatively small sample size. It is therefore possible that a difference in interoceptive awareness does exist 
between $\mathrm{SZ}$ and $\mathrm{CO}$, but that our sample lacked the statistical power to detect it. Given the important clinical implication of this preliminary finding, we encourage future studies to replicate this analysis in larger samples.

As expected, we found a significant group difference in interoceptive sensibility. SZ reported difficulty sustaining and controlling attention to, and trusting, their bodily sensations, as well as an increased tendency to worry when experiencing pain or physical discomfort. Notably, SZ also reported an increased tendency not to distract themselves from bodily sensations of pain or discomfort. These results partially contradict prior empirical and phenomenological findings. First, we note important discrepancies between our findings and Koreki et al.'s (2020), who found that SZ scored lower on Not Distracting (our results indicated the opposite effect), and higher on Noticing (we did not find a group difference on this subscale). Our findings are nonetheless largely consistent with a previous study showed that, like SZ, youths with prodromal experiences scored lower than controls on the Trusting, Not Worrying, and Attention Regulation subscales though no differences were observed in Self-Regulation and Noticing (Barbato et al., 2021). Though high-risk individuals reported enhanced abilities in Emotional Awareness and Body Listening, our findings suggest that SZ do not (though we did not observe a deficit either). Interestingly, Barbato and colleagues (2021) documented decreased scores on the Not Distracting subscale for high-risk individuals though our findings document increased in SZ. Additionally, phenomenological models of schizophrenia place hyperreflexivity (i.e., exaggerated self-awareness) at the center of the schizophrenia experience (Sass and Parnas, 2003). Thus, one would expect SZ to report exaggerated interoceptive sensibility. In sum, more research is needed to identify the specific aspects of interoceptive sensibility that might be 
disrupted or enhanced in this population. It might also be important to examine self-monitoring capacity in relation to interoceptive functions in schizophrenia. 


\section{EXPERIMENT 2: INTEROCEPTIVE FUNCTIONING AND SCHIZOTYPY}

Interoceptive functioning was examined in healthy participants in relation to schizotypy to gain further insight on the etiology and nature of interoceptive dysfunction. By studying healthy individuals with varying degrees of latent liability for schizophrenia, we also reduce potential confounding variables related to illness experience or treatment (e.g., medication effects).

Though no empirical study to date has specifically examined interoceptive functioning in schizotypal individuals, a large body of literature documents the presence of self-disturbances in individuals with elevated schizotypal traits (e.g., Asai et al., 2011, 2016; Benson et al., 2019; Cicero et al., 2017; Thakkar et al., 2011). We hypothesized that interoceptive accuracy, sensibility and awareness would be compromised in those who score high on measures of schizotypy.

\section{1. METHODS}

\section{1. 1. Participants}

109 college students were recruited from the Vanderbilt Psychology Research Subject Pool. Participants reporting substance use or alcohol abuse within the past 6 months, brain injury, neurological disease or a family history of psychosis were excluded. The Schizotypal Personality Questionnaire-Brief (SPQ-B; Raine and Benishay, 1995) was used to measure schizotypy. Participants gave written informed consent, as approved by the Vanderbilt Institutional Review Board, and were granted course credit in compensation for their participation. Demographic information of the participants is summarized in Table 2. 
Table 2. Demographic and subclinical information for Experiment 2 participants.

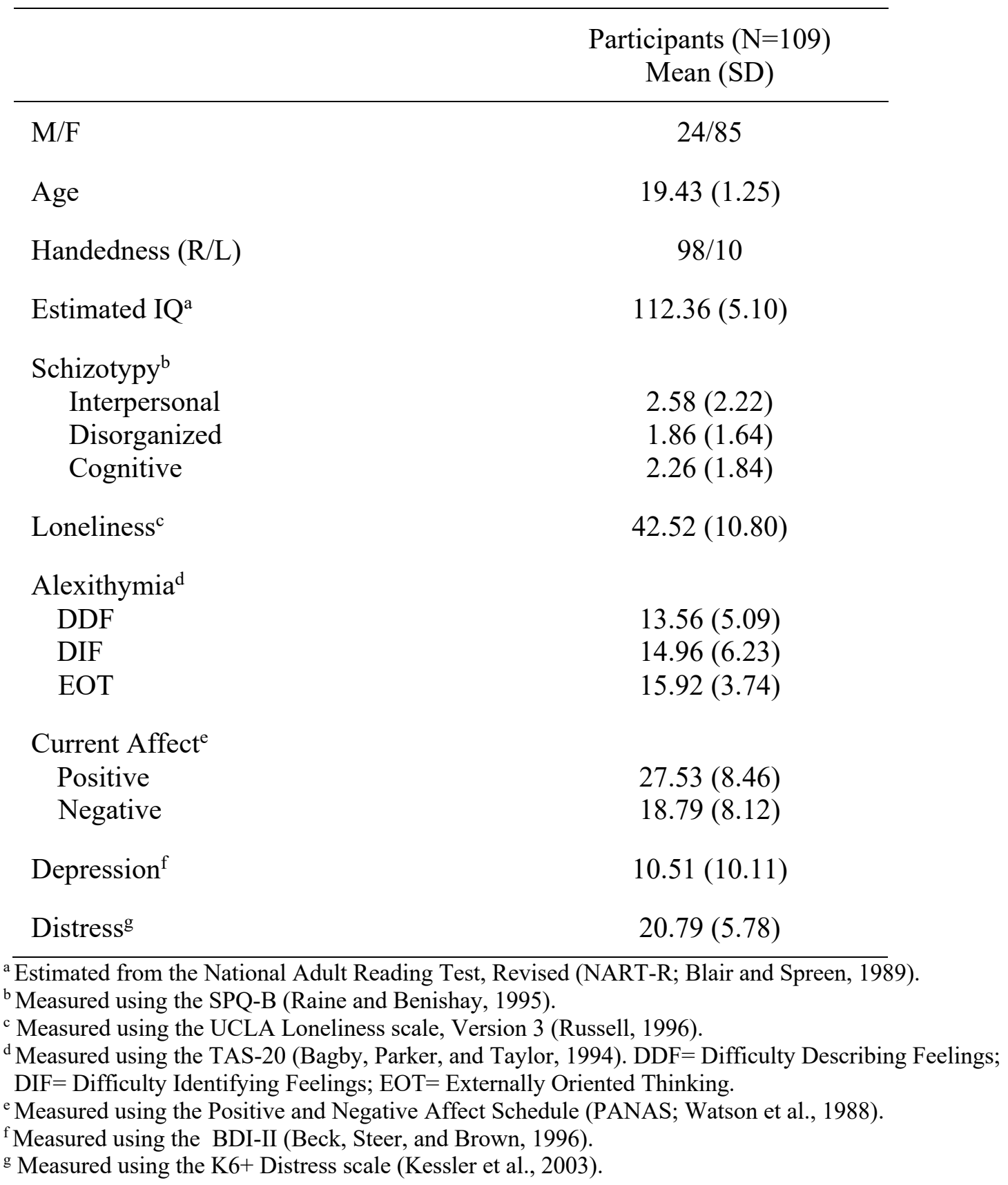




\section{1. 2. Measures and Procedure}

The procedure for Experiment 2 was identical to that of Experiment 1 except for the absence of symptoms interviews. Instead, participants completed the SPQ-B (Raine and Benishay, 1995) . Self-report measures were administered to assess loneliness (UCLA Loneliness; Russell, 1996), current affect (PANAS; Watson et al., 1988), alexithymia (TAS-20; Bagby, Parker, and Taylor, 1994), depression (BDI-II; Beck, Steer, and Brown, 1996), and distress (K6+ Distress scale; Kessler et al., 2003) as well as demographic information. Participants completed the HBCT, the MAIA, self-report measures and cognitive assessment (i.e., NART-R) in a randomized order. We note that only a subset of participants $(N=44)$ completed the MAIA.

\section{1. 3. Data Analysis}

A hierarchical regression analysis was conducted to investigate the effect of schizotypy on interoceptive accuracy. In the first model, only control variables (i.e., HR knowledge, resting HR, HRV, BMI, time perception, loneliness, current affect, alexithymia, depression, and distress) were included as predictors. SPQ-B subscales (i.e., interpersonal, cognitive, disorganized) were added to the second model, and the two models were compared using an ANOVA. Due to violations in linear regression assumptions, Spearman correlations were used to assess the relationship between interoceptive awareness and schizotypy, and a Holm correction was applied. To assess the relationship between interoceptive sensibility and schizotypy, a multivariate multiple regression was conducted. SPQ-B subscales were included as predictors and the eight MAIA dimensions as dependent variables. Finally, gender differences in interoceptive ability were assessed using Mann-Whitney $U$ tests (interoceptive accuracy and 
awareness) and MANOVA (interoceptive sensibility). The link between age and interoceptive ability was not evaluated given the limited range of ages (18-23) in this sample.

\section{2. RESULTS}

No link was found between schizotypy and interoceptive ability. A hierarchical regression analysis revealed that schizotypy did not significantly contribute to the regression model predicting interoceptive accuracy $(\Delta F=0.42, p=0.74$, See Table 3).

Table 3. Hierarchical regression models of predictors of interoceptive accuracy

\begin{tabular}{|c|c|c|}
\hline \multirow[b]{2}{*}{ Predictor variables } & \\
\hline & Model 1 & Model 2 \\
\hline Resting heart rate & $-0.27 * *$ & $-0.27^{* *}$ \\
\hline HR knowledge & -0.012 & -0.013 \\
\hline HRV & 0.07 & 0.07 \\
\hline BMI & 0.07 & 0.07 \\
\hline Time perception accuracy & $0.33 * * *$ & $0.31 * *$ \\
\hline Loneliness & 0.029 & -0.049 \\
\hline \multicolumn{3}{|l|}{ Current Affect } \\
\hline Positive & -0.046 & -0.030 \\
\hline Negative & 0.08 & 0.08 \\
\hline \multicolumn{3}{|l|}{ Alexithymia } \\
\hline DDF & -0.061 & -0.10 \\
\hline DIF & -0.054 & -0.026 \\
\hline EOT & 0.043 & 0.046 \\
\hline Depression & 0.23 & 0.26 \\
\hline Distress & 0.25 & 0.27 \\
\hline
\end{tabular}


Cognitive

Interpersonal

0.15

Disorganized

$-0.059$

Adjusted $R^{2}$

0.14

0.13

$\Delta R^{2}$

$-0.01$

$\Delta F$

0.42

$* * p<.01, * * * p<.001$

Note. This table summarizes the results of a hierarchical regression analysis which compared Model 1 (control variables) and Model 2 (control variables + schizotypy factors) in predicting interoceptive accuracy.

No link was found between interoceptive awareness and cognitive $(r(107)=-0.09$, $p=0.38)$, interpersonal, $(r(107)=-0.20, p=0.12)$, or disorganized $(r(107)=-0.10, p=0.31)$

schizotypy. Similarly, a multivariate multiple regression analysis revealed no link between schizotypy and interoceptive sensibility $(p s>0.5)$. 


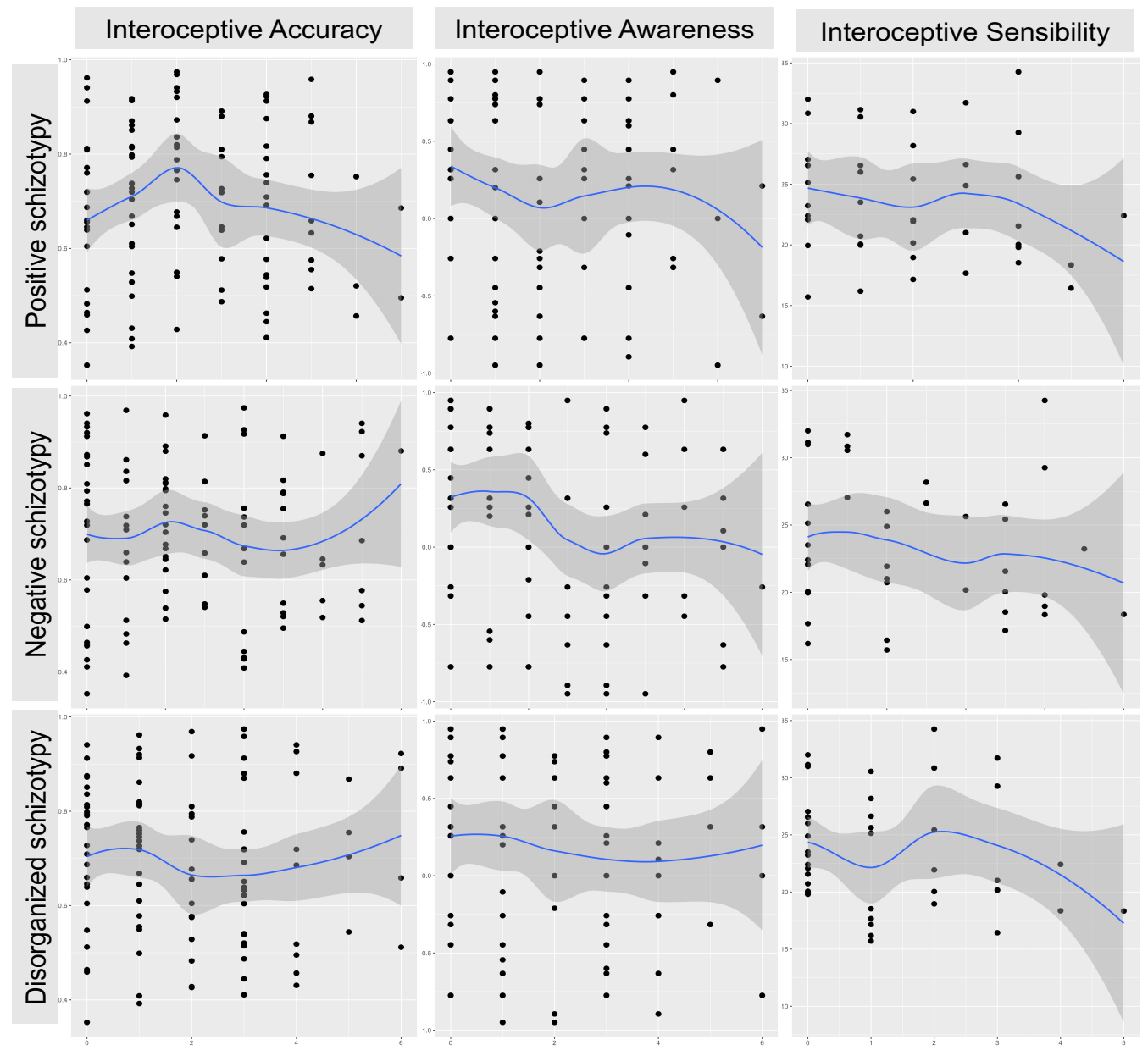

Fig. 5. Scatterplots with Loess soothing lines of schizotypy factors and interoceptive ability. The interoceptive sensibility score is an average of a participants' scores on all eight subscales on the MAIA.

Interoceptive accuracy $(\mathrm{Z}=-1.67, p=0.09)$, interoceptive awareness $(\mathrm{Z}=-0.23, p=0.81)$, and interoceptive sensibility $(F(8,35)=1.82, p=0.11)$ did not significantly differ across genders.

\section{3. DISCUSSION}

For the first time, Experiment 2 investigated interoceptive functioning in relation to schizotypy. Contrary to our predictions, there was no link between schizotypy and interoceptive ability. In fact, interoceptive accuracy, awareness, and sensibility did not systematically vary 
across any of the schizotypy dimensions. We note that the nature of our sample (i.e., healthy undergraduates) might have under-represented the higher end of the schizotypy spectrum, which limits the interpretation of our results. In fact, proponents of the categorical model of schizotypy argue that only about $10 \%$ of individuals in the general population are true "schizotypes" (Lenzenweger, 2015; Meehl, 1990). However, as previously described, we used schizotypy to assess the link between interoceptive functioning and schizophrenia liability in the general population. The gender imbalance of our sample (i.e., over-representing females and absence of non-binary and transgender individuals) further limits the generalizability of our results, though our data suggest similar interoceptive ability between males and females. Overall, our findings indicate that in the general population interceptive functioning is independent from schizotypy, though this might not be true in help-seeking prodromal individuals.

\section{GENERAL DISCUSSION}

Our work provides new insight into various aspects of interoceptive functioning in schizophrenia. Experiment 1 adds to a recent body of literature documenting low interoceptive accuracy in this population (Ardizzi et al., 2016; Koreki et al., 2020), and suggests that interoceptive dysfunction might be more pronounced in the early/acute stages of schizophrenia illness. Consistent with prior literature (Koreki et al., 2020), our results also highlighted differences in the subjective experience of interception (i.e., interoceptive sensibility) in schizophrenia, though the specific aspects of interoceptive sensibility affected remain to be clarified. 
For the first time, our study documented intact interoceptive awareness in schizophrenia, which bears important clinical implications. Interoceptive awareness refers to the metacognitive awareness of one's performance on the HBCT. Thus, SZ's intact interoceptive awareness suggests insight into their low interoceptive accuracy. Importantly, metacognitive deficits have been linked to impaired insight and poor psychosocial outcome (Lysaker et al., 2015; Lysaker et al., 2019). In contrast, SZ's insight into their interoceptive deficits might promote treatment seeking. Various psychological, physiological, and behavioral interventions aimed at strengthening the mind-body connection exist and might be beneficial to individuals with schizophrenia. For instance, Mindful Awareness in Body-Oriented Therapy (MABT) was shown to improve interoceptive processing (Price and Hoover, 2018). Another study found that an 8week body-scan practice improved interoceptive functioning (Fischer et al., 2017). In biofeedback, electrical sensors are used to increase awareness and control over one's bodily functions (e.g., heart rate). Bioelectronic medicine, which uses electrical stimulation to modulate the electrical activity of targeted brain regions, has also been proposed as a candidate for interoceptive improvements (Bonaz et al., 2021). Other experimental behavioral interventions, including the practice of power posing (Weineck et al., 2019) have been shown to improve interoceptive functioning. Increasing the attention to the self by using a photo, words, or a mirror also seems to improve interoception (Ainley et al., 2012; 2013). Physical exercise has also been shown to play a role in interoceptive functioning (Wallman-Jones and Schmidt, 2019). In a recent review paper discussing interventions and manipulations of interoception, Weng et al. (2021) argue that the practice of slow breathing might be particularly effective in training interoceptive ability. 
Experiment 2 casts new light on interoceptive functioning in the schizophrenia spectrum. Contrary to our expectations, we found no link between schizotypy and interoceptive functioning. Though our results indicate that schizotypy and interoceptive functioning are independent in the general population, this might not be true in help-seeking/prodromal populations. In fact, a recent paper documented abnormal interoceptive sensibility in youth with psychotic-like experiences (Barbato et al., 2021). Thus, it is possible that interoceptive dysfunction emerges closer to the psychosis onset, during the prodromal stage. Only a minority of individuals with high schizotypy will develop a psychotic illness (e.g., Debbané et al., 2015) and as previously mentioned, these prodromal individuals would be under-represented in our non help-seeking sample. We posit that interoceptive deficits are unrelated to schizotypy in the general population but that they may emerge during the prodromal stage preceding the onset of psychosis. However, this hypothesis needs to be tested in longitudinal studies.

There are caveats. We relied on the HBCT to measure interoceptive accuracy and awareness. The HBCT has been criticized for its methodological shortcomings (e.g., Reed et al., 1990; Zamariola et al., 2018). For instance, when asked to avoid relying on estimates, interoceptive accuracy scores are reduced by half in the HBCT (Desmedt, Lumine, and Corneille, 2018). Thus, it is possible that SZ's reduced performance on the HBCT represents differences in non-interoceptive processes rather than an interoceptive deficit. The current study attempted to address this methodological limitation by measuring various physiological and psychological variables and including them as covariates in the analyses. We note that though alternatives exists, the HBCT remains the most widely used task to measure interoceptive ability, especially in clinical populations. For instance, the Heartbeat Discrimination Task requires the participant to gauge the synchrony of their heartbeat with an external auditory stimulus (Brener 
and Kluviste, 1988). However, as Ardizzi et al. (2016) point out, the documented multisensory integration deficits in schizophrenia make this task a poor candidate for measuring interoceptive ability in this population. Thus, despite its important methodological limitations, the HBCT is better suited than alternatives for our population of interest and it allowed for replication of previous studies. We also note that the HBCT was used in combination with the MAIA to measure different aspects of interoceptive functioning such that our results do not solely rely on this task.

Overall, our findings suggest that individuals with schizophrenia perceive their internal signals with low accuracy but that they are aware of this deficit. Some subjective aspects of interoceptive experiences also appear to be dampened or heightened in schizophrenia. These differences in interoceptive functioning are not observed along the schizotypy spectrum, which suggests that interoceptive dysfunctions might only emerge during the prodromal stages. 


\section{References}

Ainley, V., Brass, M., Tsakiris, M., 2014. Heartfelt imitation: high interoceptive awareness is linked to greater automatic imitation. Neuropsychologia, 60, 21-28.

Ainley, V., Maister, L., Brokfeld, J., Farmer, H., Tsakiris, M., 2013. More of myself: Manipulating interoceptive awareness by heightened attention to bodily and narrative aspects of the self. Conscious Cogn, 22(4), 1231-1238.

Ainley, V., Tajadura-Jiménez, A., Fotopoulou, A., Tsakiris, M., 2012. Looking into myself: Changes in interoceptive sensitivity during mirror self-observation. Psychophysiol, 49(11), 1672-1676.

Andreasen, N.C., Pressler, M., Nopoulos, P., Miller, D., Ho, B.C., 2010. Antipsychotic dose equivalents and dose-years: a standardized method for comparing exposure to different drugs. Biol Psychiatry 67(3) 255-262.

Andreasen, N.C., 1983. Scale for the Assessment of Negative Symptoms. Br J Psychiatry 155(S7) 49-52.

Andreasen, N.C., 1989. Scale for the Assessment of Positive Symptoms. Br J Psychiatry 155(S7) 49-55.

Ardizzi, M., Ferri, F., 2018. Interoceptive influences on peripersonal space boundary. Cogn, 177, 79-86.

Ardizzi, M., Ambrosecchia, M., Buratta, L., Ferri, F., Peciccia, M., Donnari, S., ... Gallese, V., 2016. Interoception and positive symptoms in schizophrenia. Front Hum Neurosci, 10, 379.

Asai, T., Kanayama, N., Imaizumi, S., Koyama, S., Kaganoi, S., 2016. Development of embodied sense of self scale (ESSS): exploring everyday experiences induced by anomalous self-representation. Front Psychol, 7, 1005.

Asai, T., Mao, Z., Sugimori, E., Tanno, Y., 2011. Rubber hand illusion, empathy, and schizotypal experiences in terms of self-other representations. Conscious Cogn, 20(4), 1744-1750.

Bagby, R. M., Parker, J. D. A., Taylor, G. J., 1994. The twenty-item Toronto alexithymia scale: I. item selection and cross-validation of the factor structure. J Psychosom Res, 38(1), 23 32 .

Barbato, M., Arora, T., Al Hemeiri, S., AlJassmi, M. A., 2021. Looking within: Interoceptive sensibility in young adults with psychotic-like experiences. Early Interv Psychiatry Early Interv Psychiatry. 
Beck, A.T., Steer, R.A., Brown, G.K., 1996. Manual for the Beck Depression Inventory-II. San Antonio, TX: Psychological Corporation.

Benson, T. L., Brugger, P., Park, S., 2019. Bodily self-disturbance in schizophrenia-spectrum populations: Introducing the Benson et al. Body Disturbances Inventory (B-BODI). PsyCh journal, 8(1), 110-121.

Blair, J.R., Spreen, O., 1989. Predicting premorbid IQ: a revision of the National Adult Reading Test. Clin Neuropsychol. 3(2) 129-136.

Bleuler, E. Dementia Praecox ou Groupe des Schizophrénies (Epel Editions, Paris, 1993) (1st publication 1911).

Bonaz, B., Lane, R. D., Oshinsky, M. L., Kenny, P. J., Sinha, R., Mayer, E. A., Critchley, H. D., 2021. Diseases, disorders, and comorbidities of interoception. Trends Neurosci, 44(1), $39-51$.

Brener, J., Kluvitse, C., 1988. Heartbeat detection: judgments of the simultaneity of external stimuli and heartbeats. Psychophysiol, 25(5), 554-561.

Cicero, D. C., Neis, A. M., Klaunig, M. J., Trask, C. L., 2017. The Inventory of Psychotic-Like Anomalous Self-Experiences (IPASE): Development and validation. Psychol Assess, 29(1), 13.

Cohen, S., Kamarck, T., Mermelstein, R., 1983. A global measure of perceived stress. Journal of health and social behavior, 385-396.

Craig, A. D., 2002. How do you feel? Interoception: the sense of the physiological condition of the body. Nat Rev Neurosci, 3(8), 655-666.

Damasio, A. R., 2012. Self comes to mind: Constructing the conscious brain. Vintage.

Debbané, M., Eliez, S., Badoud, D., Conus, P., Flückiger, R., Schultze-Lutter, F., 2015. Developing psychosis and its risk states through the lens of schizotypy. Schizophr Bull, 41(suppl_2), S396-S407.

Desmedt, O., Luminet, O., Corneille, O., 2018. The heartbeat counting task largely involves noninteroceptive processes: Evidence from both the original and an adapted counting task. Biol Psychol, 138,.185-188.

Ferroni, F., Ardizzi, M., Sestito, M., Lucarini, V., Daniel, B.D., Paraboschi, F., Tonna, M., Marchesi, C. and Gallese, V., 2019. Shared multisensory experience affects Others' boundary: The enfacement illusion in schizophrenia. Schizophr Res, 206, 225-235.

Filippetti, M. L., Tsakiris, M., 2017. Heartfelt embodiment: Changes in body-ownership and self-identification produce distinct changes in interoceptive accuracy. Cogn, 159, 1-10. 
First, M. B., Williams, J. B. W., Karg, R. S., Spitzer, R. L., 2015. Structured clinical interview for DSM-5-Research version (SCID-5 for DSM-5, research version; SCID-5-RV). Arlington, VA: American Psychiatric Association, 1-94.

Fischer, D., Messner, M., Pollatos, O., 2017. Improvement of interoceptive processes after an 8week body scan intervention. Front Hum Neurosci, 11, 452.

Garfinkel, S. N., Seth, A. K., Barrett, A. B., Suzuki, K., Critchley, H. D., 2015. Knowing your own heart: distinguishing interoceptive accuracy from interoceptive awareness. Biol Psychol, 104, 65-74.

Grant, P., Munk, A.J.L, Kuepper, Y., Wielpuetz, C., \& Hennig, J., 2015. Additive Genetic Effects for Schizotypy Support a Fully-Dimensional Model of Psychosis-Proneness. J Individ Differ, 36, 87-92.

Hur, J. W., Kwon, J. S., Lee, T. Y., Park, S., 2014. The crisis of minimal self-awareness in schizophrenia: a meta-analytic review. Schizophr Res, 152(1), 58-64.

Kean, C., 2009. Silencing the self: schizophrenia as a self-disturbance. Schizophr Bull, 35(6), 1034.

Kessler, R. C., Barker, P. R, Colpe, L. J., Epstein, J. F., Gfroerer, J. C., Hiripi, E., Howes, M.J., Normand, S.L., Manderscheid, R. W., Walters, E. E., Zaslavsky, A. M., 2003. Screening for serious mental illness in the general population. Arch Gen Psychiatry, 60(2), 184-9.

Khalsa, S. S., Rudrauf, D., Tranel, D., 2009. Interoceptive awareness declines with age. Psychophysiol, 46(6), 1130-1136.

Knapp-Kline, K., Kline, J. P., 2005. Heart rate, heart rate variability, and heartbeat detection with the method of constant stimuli: slow and steady wins the race. Biol Psychol, 69(3), 387396.

Koreki, A., Funayama, M., Terasawa, Y., Onaya, M., Mimura, M., 2021. Aberrant interoceptive accuracy in patients with schizophrenia performing a heartbeat counting task. Schizophr Bull Open, 2(1), sgaa067.

Lenzenweger, M. F. 2015. Thinking clearly about schizotypy: hewing to the schizophrenia liability core, considering interesting tangents, and avoiding conceptual quicksand. Schizophr Bull, 41(suppl_2), S483-S491.

Lysaker, P. H., Dimaggio, G., 2014. Metacognitive capacities for reflection in schizophrenia: implications for developing treatments. Schizophr Bull, 40(3), 487-491.

Lysaker, P. H., Gagen, E., Wright, A., Vohs, J. L., Kukla, M., Yanos, P. T., Hasson-Ohayon, I., 2019. Metacognitive deficits predict impaired insight in schizophrenia across symptom profiles: a latent class analysis. Schizophr Bull, 45(1), 48-56. 
Lysaker, P. H., Vohs, J., Minor, K. S., Irarrázaval, L., Leonhardt, B., Hamm, J. A., ... Dimaggio, G., 2015. Metacognitive deficits in schizophrenia: presence and associations with psychosocial outcomes. J Nerv Ment Dis, 203(7), 530-536.

Meehl, P.E., 1962. Schizotaxia, schizotypy, schizophrenia. Am Psychol, 17, 827- 838.

Meehl, P. E. 1990. Toward an integrated theory of schizotaxia, schizotypy, and schizophrenia. J Pers Disord, 4(1), 1-99.

Mehling, W. E., Price, C., Daubenmier, J. J., Acree, M., Bartmess, E., Stewart, A., 2012. The multidimensional assessment of interoceptive awareness (MAIA). PloS one, 7(11), e48230.

Michael, J., Park, S. 2016. Anomalous bodily experiences and perceived social isolation in schizophrenia: an extension of the social deafferentation hypothesis. Schizophr Res, 176(2-3), 392-397.

Nelson, B., Thompson, A., Yung, A. R., 2012. Basic self-disturbance predicts psychosis onset in the ultra-high risk for psychosis "prodromal" population. Schizophr Bull, 38(6), 12771287.

Palmer, C. E., Tsakiris, M., 2018. Going at the heart of social cognition: is there a role for interoception in self-other distinction?. Curr Opin Psychol, 24, 21-26.

Park, S., Nasrallah, H. A., 2014. The varieties of anomalous self experiences in schizophrenia: Splitting of the mind at a crossroad. Schizophr Res, 152, 1-4.

Price, C. J., Hooven, C., 2018. Interoceptive awareness skills for emotion regulation: Theory and approach of mindful awareness in body-oriented therapy (MABT). Front Psychol, 9, 798.

Quigley, K. S., Kanoski, S., Grill, W. M., Barrett, L. F., Tsakiris, M., 2021. Functions of interoception: from energy regulation to experience of the self. Trends Neurosci, 44(1), 29-38.

Raballo, A., Poletti, M., Preti, A., Parnas, J., 2021. The Self in the Spectrum: A Meta-analysis of the Evidence Linking Basic Self-Disorders and Schizophrenia.

Raine, A., Reynolds, C., Lencz, T., Scerbo, A., Triphon, N., \& Kim, D., 1994. Cognitiveperceptual, interpersonal, and disorganized features of schizotypal personality. Schizophr Bull, 20(1), 191-201.

Raine, A., Benishay, D., 1995. The SPQ-B: A brief screening instrument for schizotypal personality disorder. J Pers Disord, 9(4), 346-355. 
Reed, S.D., Harver, A., Katkin, E.S., 1990. Interoception. In J.T. Cacioppo L.G.Tassinary (Eds.), Principles of Psychophysiol : Physical, social, and inferential elements (pp.253291). Cambridge, UK: Cambridge University Press.

Ring, C., Brener, J., 1996. Influence of beliefs about heart rate and actual heart rate on heartbeat counting. Psychophysiol, 33(5), 541-546.

Rouse, C. H., Jones, G. E., Jones, K. R., 1988. The effect of body composition and gender on cardiac awareness. Psychophysiol, 25(4), 400-407.

Russell, D., 1996. UCLA Loneliness Scale (Version 3): Reliability, validity, and factor structure. J Pers Assess, 66(1), 20-40.

Saks, E. R., 2007. The center cannot hold: My journey through madness. Hachette UK.

Sandsten, K.E., Nordgaard, J., Kjaer, T.W., Gallese, V., Ardizzi, M., Ferroni, F., Petersen, J. and Parnas, J., 2020. Altered self-recognition in patients with schizophrenia. Schizophr Res, 218, 116-123.

Sass, L. A., Parnas, J., 2003. Schizophrenia, consciousness, and the self. Schizophr Bull, 29(3), 427-444.

Schandry, R., 1981. Heart beat perception and emotional experience. Psychophysiol, 18(4), 483488.

Shaffer, F., Ginsberg, J. P., 2017. An overview of heart rate variability metrics and norms. Front Public Health, 5, 258.

Suzuki, K., Garfinkel, S. N., Critchley, H. D., Seth, A. K., 2013. Multisensory integration across exteroceptive and interoceptive domains modulates self-experience in the rubber-hand illusion. Neuropsychologia, 51(13), 2909-2917.

Tajadura-Jiménez, A., Tsakiris, M., 2014. Balancing the "inner" and the "outer" self: Interoceptive sensitivity modulates self-other boundaries. J Exp Psychol Gen, 143(2), 736.

Thakkar, K. N., Nichols, H. S., McIntosh, L. G., Park, S., 2011. Disturbances in body ownership in schizophrenia: evidence from the rubber hand illusion and case study of a spontaneous out-of-body experience. PloS one, 6(10), e27089.

Torregrossa, L.J., Snodgress, M.A., Hong, S.J., Nichols, H.S., Glerean, E., Nummenmaa, L., Park, S., 2019. Anomalous bodily maps of emotions in schizophrenia. Schizophr Bull, 45(5), 1060-1067.

Tsakiris, M., 2017. The multisensory basis of the self: from body to identity to others. Q J Exp Psychol, 70(4), 597-609. 
Tsakiris, M., Jiménez, A. T., Costantini, M., 2011. Just a heartbeat away from one's body: interoceptive sensitivity predicts malleability of body-representations.Proc R Soc B Biol Sci, 278(1717), 2470-2476.

Ventura, J., Lukoff, D., Nuechterlein, K. H., Liberman, R. P., Green, M., Shaner, A., 1993. Appendix 1: Brief Psychiatric Rating Scale (BPRS) expanded version (4.0) scales, anchor points and administration manual. Int J Methods Psychiatr Res, 3(227), 43.

Wallman-Jones., A. Schmidt., M., 2019. Physical Activity to Improve Interoception and Emotional Regulation: Theoretical Considerations for Future Research. In: SGS 11TH Annual meeting of the Swiss Society of Sport Science, Fribourg, Switzerland.

Watson, D., Clark, L. A., Tellegen, A., 1988. Development and validation of brief measures of positive and negative affect: the PANAS scales. J Pers Soc Psychol, 54(6), 1063.

Weineck, F., Messner, M., Hauke, G., Pollatos, O., 2019. Improving interoceptive ability through the practice of power posing: A pilot study. PloS One, 14(2), e0211453.

Weng, H. Y., Feldman, J. L., Leggio, L., Napadow, V., Park, J., Price, C. J., 2021. Interventions and manipulations of interoception. Trends Neurosci, 44(1), 52-62.

Zamariola, G., Maurage, P., Luminet, O., Corneille, O., 2018. Interoceptive accuracy scores from the heartbeat counting task are problematic: Evidence from simple bivariate correlations. Biol Psychol, 137, 12-17. 